\title{
Neutral and lonic Particle Emission Produced by Laser Irradiation of a GaAs Surface
}

\author{
G. R. MÖHLMANN
}

Akzo Research Laboratories Arnhem, Corporate Research Department, Postbox 60, 6800 AB Arnhem, The Netherlands

(Received 28 October 1983; in final form 1 February 1984)

The emission of gas phase particles from a GaAs surface, due to irradiation at $1064 \mathrm{~nm}$ by an unfocussed Q-switched Nd:YAG laser beam, has been studied. Mass spectra have been recorded with the aid of a quadrupole mass spectrometer, and the ion-neutral ratio of the emitted particles has been measured with an electrical diode set up. The applied incident laser radiation power density was varied in the range 1.2-9.6 $\times 10^{7}$ Watts $/ \mathrm{cm}^{2}$ and was delivered as $10 \mathrm{~ns}(\mathrm{fwhm})$ long pulses. It appeared that mainly Ga atoms and $\mathrm{As}_{2}$ molecules were emitted, together with minor amounts $(<1 \%)$ of $\mathrm{Ga}_{2}$ and $\mathrm{GaAs}$ molecules. Besides the emission of neutral particles, the formation of $\mathrm{Ga}^{+}$ atomic ions was observed. By measuring the ratio of $\mathrm{Ga}^{+}$ions and $\mathrm{Ga}$ neutral atoms it could be shown that the (ionic) particle emission is governed by a thermal mechanism for $1064 \mathrm{~nm}$ incident radiation. This latter experimental result differs from that obtained if a ruby laser $(694 \mathrm{~nm})$ is used as the radiation source.

\section{INTRODUCTION}

It is known that high power laser radiation incident on an absorbing surface can melt the upper layer or even remove particles such as electrons, neutrals or ions from that surface. ${ }^{1}$ Practical applications of these phenomena are laser annealing of ion implanted semiconductors as well as hardening, cutting or drilling of a variety of materials.

In the case of Q-switched laser irradiation/annealing of a GaAs surface with unfocussed laser beams there appeared to be insufficient experimental data about the amounts and types of species that are removed from the surface during and after the irradiation process. ${ }^{2}$ 
Thus far only experiments have been carried out on GaAs with (normal pulse) focussed ruby laser beams $\left(10^{7}\right.$ Watts $\left./ \mathrm{cm}^{2}, 1 \mathrm{~ms}, 3-5 \mathrm{~J} / \mathrm{pulse}^{3,4}\right)$ and with tightly focussed Q-switched Nd:YAG laser light $\left(10^{9}\right.$ Watts $/ \mathrm{cm}^{25}$ ).

The experimental results described in Refs. 3 and 4 suggest that, for a ruby laser light flux density of only $10^{7}$ Watts $/ \mathrm{cm}^{2}$ at $694 \mathrm{~nm}$, a very large fraction of the emitted $\mathrm{Ga}$ particles is already in the ionic state as they leave the surface, thus indicating a non-thermal ionneutral ratio.

In the present work the generation of gas phase particles up to 200 a.m.u., due to GaAs surface irradiation with Q-switched unfocussed Nd:YAG laser light at $1064 \mathrm{~nm}$, is described. The used laser radiation exhibits an uniform intensity distribution across the beam. The applied power densities are in the range 1.2-9.6 $\times$ $10^{7}$ Watts $/ \mathrm{cm}^{2}$.

Simultaneously with the present work, experiments with unfocussed Q-switched ruby laser light on GaAs have been performed and the $\mathrm{Ga}$ and As emission yields have been determined via RutherfordBack-Scattering. ${ }^{6}$

\section{EXPERIMENTAL}

The apparatus used in the present experiment (see Figure 1) consists of a quadrupole mass spectrometer (range 1-200 a.m.u.) equipped with an open ionization chamber. Through this chamber a laser beam and an (ionizing) electron beam $(0-100 \mathrm{eV})$ are directed at right angles to each other. The spectrometer set up has been installed in a vacuum chamber (pressure about $1 \times 10^{-7} \mathrm{P}$ ) which is equipped with a sapphire window for the transmission of the laser light. A target can be placed near the ionization chamber such that it is hit by the laser beam and the emitted (and successively ionized) particles can enter the mass filter. The mass resolved species are detected by an electron multiplier with a gain up to about $10^{6}$. The multiplier signal is converted and amplified to $10^{4}-10^{10}$ Volts/Ampere and fed into a boxcaraverager/gated-integrator which detection window (time gate) is controlled by a variable delay signal synchronized to the laser pulse. Therefore, all presented data represent average values due to multiple laser pulses. Simultaneously the electron multiplier signal can be 


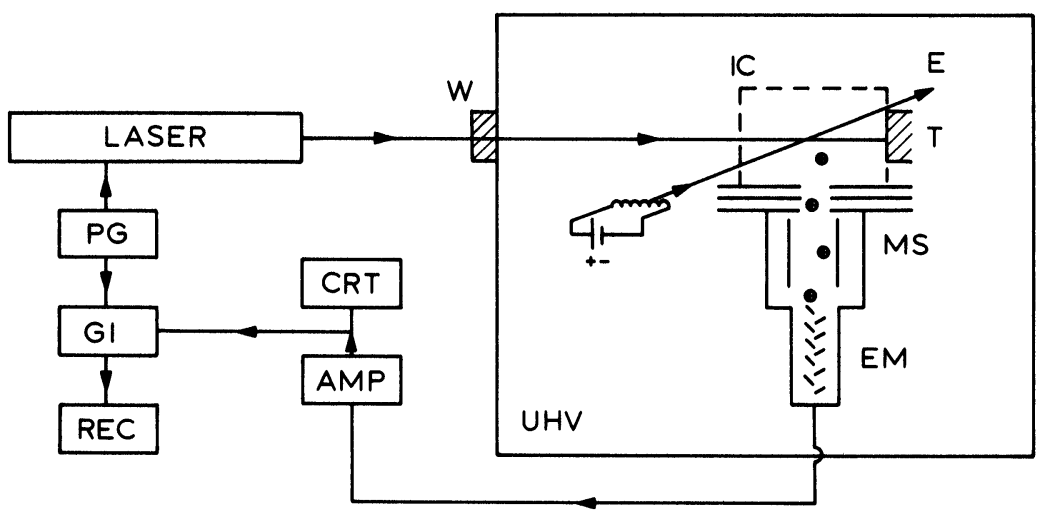

FIGURE 1 Schematic view of the experimental set up in which $\mathrm{W}$ means window; IC: ionization chamber; E: electron beam; T: target; MS: mass spectrometer; EM: electron multiplier; UHV: vacuum chamber; AMP: amplifier; CRT: oscilloscope; GI: gated integrator; PG: pulse generator; REC: recorder.

displayed on an oscilloscope for easy alignment and optimization of the experimental set up.

By scanning the mass range with a speed of about 1 a.m.u./s a time discriminated mass spectrum is recorded which only reflects the laser induced contribution to the experimental data and thus is free from disturbing (non laser related) contributions. The width of the detection window is chosen such $(50-150 \mu \mathrm{s}$.) that for a particular mass all particles are detected that are generated due to the laser influence on the target.

By switching the ionizing electron beam current on and off one can get an impression whether all emitted particles of a particular mass are neutral or that a fraction of them has already been ionized due to the laser action. It is estimated that under the applied experimental circumstances the electron beam can ionize a fraction up to about $10^{-5}$ of the neutrals present; the exact fraction depends (among others) on the ionization cross section of the species under study which is different for different species. Variation of the kinetic energy of the ionizing electrons gives insight whether the detected ions are formed via a primary ionization process or via a secondary dissociative ionization process as discussed further on.

For the determination of the ion-neutral ratio of the laser emitted particles a simple diode set up has been used. This consisted of an 
anode (kept at $+500 \mathrm{~V}$ ) containing the GaAs target, and of a cathode (connected to earth via the $50 \Omega$ input channel of the boxcar averager/gated-integrator) which is placed in such a way that most of the emitted particles are collected. The laser beam hits the GaAs target at an angle of about $45^{\circ}$. The emitted charge wave form is recorded by scanning the time window of the boxcar-averager with respect to the laser pulse; successive integration yields the total average emitted charge per laser pulse. Weighting the cathode before and after the experiment ( 36000 pulses in 1 hour) gives the total amount (ion + neutral) of emitted species, thus enabling one to calculate the ion-neutral ratio.

A uniform spatial distribution of the intensity in the laser beam at $1064 \mathrm{~nm}$ has been applied for the experiments. Such a distribution is obtained by running the oscillator of the Nd:YAG laser system at maximum power and adjusting the desired output power by varying the amplifier pump (lamp) energy. Saturation of the amplification at the center of the beam caused a nearly flat intensity profile over the central $3 \mathrm{~mm}$ cross section of the beam. Applying a $3 \mathrm{~mm}$ pinhole just in front of the sapphire entrance window resulted in an almost uniform intensity distribution with a variation of less than $\pm 5 \%$. For the determination of the incident power density $(\mathrm{P})$ at $1064 \mathrm{~nm}$ the laser pulse is approximated (taking into account the actual temporal pulsc shape) by a square pulse ( $10 \mathrm{~ns}$ wide) containing 0.7 of the total energy (E) and thus leading to the relation $\mathrm{P}=\mathrm{E} \times 0.7 / 10^{-8}$ Watts $/ \mathrm{cm}^{2}$.

\section{RESULTS}

In Figure 2 the mass spectra are presented which have been obtained if a GaAs surface is hit by laser beams of various power densities. For each applied power density three spectra are shown, namely:

a spectrum (el) due to electron impact of the species in the ionization chamber only (no laser beam present);

a spectrum $(\mathrm{h} \nu)$ which reflects the direct formation of ions by laser radiation only (no ionizing electrons present);

a spectrum $(\mathrm{h} \nu+\mathrm{el})$ in which the combined contribution of direct ionization (by $h \nu$ ) and neutral formation (by $h \nu$, but successively ionized by electrons) is shown. 

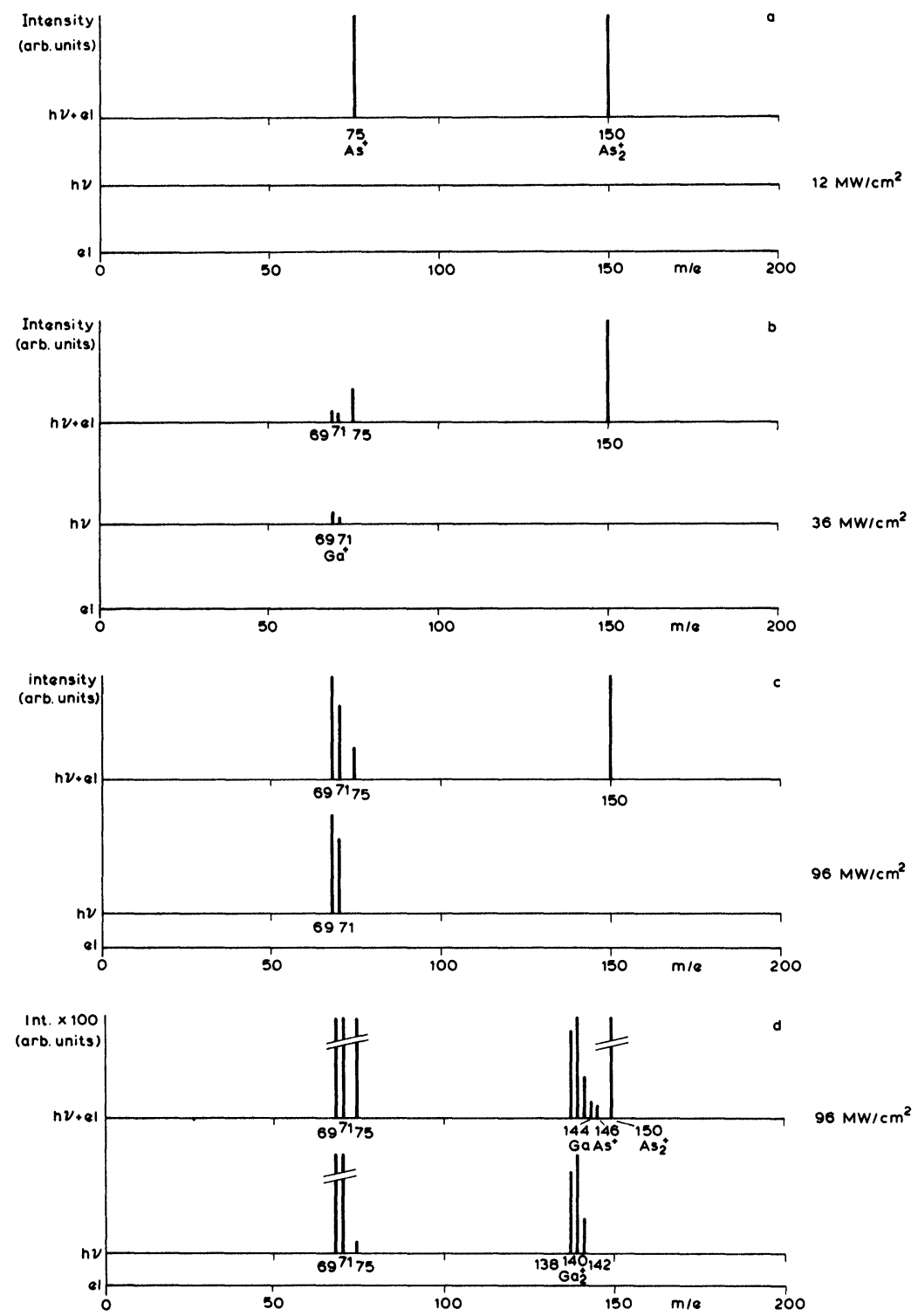

FIGURE 2 Mass spectra obtained at various power densities of laser radiation. (a) $12 \mathrm{MW} / \mathrm{cm}^{2}$; (b) $36 \mathrm{MW} / \mathrm{cm}^{2}$; (c) $96 \mathrm{MW} / \mathrm{cm}^{2}$, and (d) $100 \times$ enlarged intensity scale of spectrum $\mathrm{c}$. 
Comparison of the three spectra gives an indication whether the emitted particles are mainly neutral or do contain already a measurable ionized fraction as they leave the surface. It is clear from the pictures that all observed emitted particles are due to laser action because no signal is observed if only the electron beam is present. The peaks in the mass spectra have been assigned to the following species: $\mathrm{Ga}^{+}$at $\mathrm{m} / \mathrm{e}=69$ and $71 ; \mathrm{As}^{+}$at $\mathrm{m} / \mathrm{e}=75 ; \mathrm{Ga}_{2}^{+}$at $\mathrm{m} / \mathrm{e}=138,140$ and 142 ; $\mathrm{GaAs}^{+}$at $\mathrm{m} / \mathrm{e}=144$ and $146 ; \mathrm{As}_{2}^{+}$at $\mathrm{m} / \mathrm{e}=150$. The following conclusions can be drawn from the spectra in Figure 2, namely:

At the lowest $\mathrm{P}$ applied $\left(1.2 \times 10^{7}\right.$ Watts $\left./ \mathrm{cm}^{2}\right)$ only neutral arsenic species are formed. The $\mathrm{Ga}$ contribution is less than $1 \%$ in comparison to $\mathrm{As}_{2}$. The $\mathrm{As}^{+}$peak is due to dissociative ionization by electron impact of neutral $\mathrm{As}_{2}$ as explained further on.

At moderate $\mathrm{P}\left(3.6 \times 10^{7} \mathrm{Watts} / \mathrm{cm}^{2}\right)$ only $\mathrm{Ga}$ particles and $\mathrm{As}_{2}$ molecular neutrals are detected. The intensities of the $\mathrm{Ga}^{+}$signals in the " $\mathrm{h} \nu$ " and the " $\mathrm{h} \nu+\mathrm{el}$ " spectra are about equal indicating that the $\mathrm{Ga}^{+}$contribution is at least about $10^{-5}$ of the total $\mathrm{Ga}$ emission. The $\mathrm{As}^{+}$peak is again due to dissociative ionization of $\mathrm{As}_{2}$. At the highest $P$ applied $\left(9.6 \times 10^{7}\right.$ Watts $\left./ \mathrm{cm}^{2}\right)$ mainly $\mathrm{Ga}^{+}$and $\mathrm{Ga}$ particles together with $\mathrm{As}_{2}$ molecular neutrals are formed as in the former experiment. However, at this power density some additional species such as directly ionized $\mathrm{As}^{+}$and $\mathrm{Ga}_{2}^{+}$, together with $\mathrm{GaAs}$ neutrals are present in minor quantities $(<1 \%)$ as shown in the " $100 \times$ " spectra.

It was noticed, by varying the kinetic energy of the ionizing electrons, that the appearance potential of $\mathrm{As}^{+}$in the mass spectrometer was about $4 \mathrm{eV}$ higher than that of $\mathrm{As}_{2}^{+}$. Taking into account the dissociation energy of $\mathrm{As}_{2}$ (equal to $3.81 \mathrm{eV}^{7}$ ), and the fact that the ionization potentials of most atoms are often not more than $0.5 \mathrm{eV}$ higher than those of their corresponding dimers ${ }^{7}$, this indicates that $\mathrm{As}^{+}$is formed via dissociative ionization of $\mathrm{As}_{2}$. So, only $\mathrm{As}_{2}$ neutral molecules initially result (together with $\mathrm{Ga}^{+}$and $\mathrm{Ga}$ particles) from laser irradiation of solid GaAs, and no As atoms.

Comparison of the present results at $1064 \mathrm{~nm}$ with those obtained at $694 \mathrm{~nm}$ with focussed (normal pulse) ruby laser light $\left(10^{7}\right.$ Watts $/ \mathrm{cm}^{2}, 1 \mathrm{~ms}$ pulse $\left.\mathrm{c}^{3,4}\right)$, shows that the mass spectra exhibit common features. However, an important difference is that with the ruby laser a much larger fraction of the $\mathrm{Ga}$ particles is ionized and that more atomic than molecular arsenic is found than in the present 
Q-switched Nd:YAG laser experiment. The reason for this difference might be the shorter wavelength of the ruby laser light, combined with the absorption characteristics of $\mathrm{GaAs}$ and of the produced $\mathrm{As}_{2}$.

The diode set up experiments have been carried out with a laser pulse energy of 0.096 Joule which corresponds to a power density of $9.6 \times 10^{7}$ Watts $/ \mathrm{cm}^{2}$ for a $0.3 \mathrm{~cm}$ diameter laser beam. The resulting emitted charge per laser pulse equalled $2.0( \pm 1.0) \times 10^{-7}$ Coulombs $\left(=1.3( \pm 0.6) \times 10^{12}\right.$ ions $)$, and the total transported mass per pulse was determined to be $8.5( \pm 2.0) \times 10^{-7}$ grams $\left(=3.5( \pm 0.8) \times 10^{15}\right.$ particles $\mathrm{GaAs}$ ). The charged particles consist predominantly of $\mathrm{Ga}^{+}$ atomic ions (as can be seen in the mass spectra of Figure 2) so that the above measured values yield the ion-neutral ratio for atomic gallium which is calculated to be equal to $3.7( \pm 2.5) \times 10^{-4}$.

\section{DISCUSSION}

Several features observed in the mass spectra, or which are relevant for their interpretation, will be discussed in the following sections, namely:

(a) The estimated surface temperatures reached at the three applied power densities at $1064 \mathrm{~nm}$.

(b) The dominance of the formation of atomic gallium and molecular arsenic in the primary laser process in comparison to the formation of molecular gallium, gallium arsenide and atomic arsenic.

(c) The fractional ionization of the gallium atoms and the absence of ionization in the case of molecular arsenic.

ad a

The thermal emission of neutral and ionic particles from a surface strongly depends on the temperature of that surface. The emission rate increases with increasing temperature. Therefore the surface temperatures are estimated for the three applied power densities and are used in the following section for the explanation of the observed mass spectra. Irradiating a GaAs surface with a $20 \mathrm{~ns}$ long (Qswitched) ruby laser pulse, exhibiting an energy density of $1 \mathrm{Joule} / \mathrm{cm}^{2}$, results in a calculated temperature rise of about $2500 \mathrm{~K} .{ }^{6}$ In the present 
experiment the maximum applied pulse energy density equals about $1.4 \mathrm{~J} / \mathrm{cm}^{2}$ deposited in about $10 \mathrm{~ns}$. For the temperature rise estimation in this case one has to consider that at $1064 \mathrm{~nm} \mathrm{GaAs}$ has a low absorption coefficient at room temperature $\left(30 \mathrm{~cm}^{-1}\right)$. However, it is assumed that this initial transparency will disappear on a short time scale due to rapid population of the conduction band by electrons from the valence band. This rapid population is a consequence of free carrier absorption, avalanche multiplication and multi-photon absorption by valence band electrons. So, during the laser pulse the absorption coefficient increases drastically due to the above mentioned features. ${ }^{8}$ For the estimates of the surface temperatures it is thus assumed that the initial transparency does not play a decisive role and that the temperature rise is roughly proportional to the deposited energy density. Applying the calculated temperature rise of $2500 \mathrm{~K}$ for $1 \mathrm{~J} / \mathrm{cm}^{2}$ radiation, it has been derived that for incident power densities of $1.2 \times 10^{7}$ and $3.6 \times 10^{7}$ Watts $/ \mathrm{cm}^{2}\left(0.17\right.$ and $0.51 \mathrm{~J} / \mathrm{cm}^{2}$ respectively) the surface temperatures becomes about $700 \mathrm{~K}$ and $1600 \mathrm{~K}$ respectively. At $9.6 \times 10^{7}$ Watts $/ \mathrm{cm}^{2}\left(=1.4 \mathrm{~J} / \mathrm{cm}^{2}\right)$ the surface temperature fairly reaches the boiling point of $\mathrm{Ga}(2676 \mathrm{~K})$. It is stated that the obtained temperatures are not more than rough estimates and will be only used further on for a qualitative explanation of the obtained mass spectra.

ad b

The experimental observation of the appearance of molecular arsenic, and at higher power densities also that of atomic gallium, can be explained using the partial pressures diagram of the GaAs system. ${ }^{9}$ For low temperatures (power density) the diagram shows that the vapour pressure of arsenic molecules is about 6 orders of magnitude larger than that of gallium. Irradiation of $\mathrm{GaAs}$ at low power density $\left(1.2 \times 10^{7} \mathrm{Watts} / \mathrm{cm}^{2}\right)$ thus yields gaseous molecular arsenic causing a gallium enrichment of the surface layer. Eventually produced gaseous $\mathrm{As}_{4}$ particles could not be detected in the present experiment due to the limited mass range of the applied mass spectrometer. However, according to the diagram, ${ }^{9}$ for gallium enriched $\mathrm{GaAs}$ the $\mathrm{As}_{2}$ vapour pressure is about 100 times higher than that of $\mathrm{As}_{4}$ at lower temperature. For higher power densities (temperatures) the current arsenic depletion is considerable and the gallium enriched surface 
layer consequently emits an increasing fraction of gallium particles. This effect is seen at the power densities equal to $3.6 \times 10^{7}$ and $9.6 \times 10^{7} \mathrm{Watts} / \mathrm{cm}^{2}$. The absence of atomic arsenic, molecular gallium and molecular gallium arsenide is due to the fact that their existence is thermodynamically not favoured under the experimental circumstances.

\section{ad C}

The ratio $\mathrm{R}$ of the thermal emissions of ions and their corresponding neutrals is given by the so called Langmuir-Saha equation ${ }^{10}$ :

$$
R=\left(g_{+} / g_{0}\right) \exp ((\phi-I) / k T)
$$

in which $g_{+}$and $g_{0}$ are the degeneracies of the ionic and neutral states respectively, $\phi$ the electron work function, $I$ the ionization potential and $k$ the Boltzmann constant. In the case of gallium: $g_{+} / g_{0}=\frac{1}{2}$, $I=6 \mathrm{eV}, k=8.6 \times 10^{-5} \mathrm{eV} / \mathrm{K}$ and $T=2676 \mathrm{~K}$. Inserting the $\mathrm{GaAs}$ value $\phi=4.35 \mathrm{eV}^{11}$ the thermal ion-neutral ratio equals $3.8 \times 10^{-4}$. This is in agreement with our experimentally obtained ratio. For arsenic where $I$ equals about $9.5 \mathrm{eV}$ the $R$ value is much smaller $\left(\sim 10^{-10}\right)$. So, this calculation explains the absence of initially ionized arsenic in the gas phase and confirms the thermal character of the particle emission process for $1064 \mathrm{~nm}$ laser radiation on $\mathrm{GaAs}$.

\section{Acknowledgements}

The author likes to thank Prof. F. W. Saris and Prof. J. Los of the FOM Institute at Amsterdam for their interest and discussions concerning this subject.

\section{References}

1. G. F. Ready, Effects of High-Power Laser Radiation (Academic Press, New York, London, 1971).

2. Z. L. Wang and F. W. Saris, Phys. Letters 83 A7, 367 (1981).

3. V. P. Zakharov and I. M. Protas, Bull. Acad. Scie. USSR Phys. Rev. 38, 51 (1974).

4. V. P. Zakharov and I. M. Protas, Sov. Phys. Techn. Phys. 17, 528 (1972).

5. A. I. Busygin, Sov. Techn. Phys. Letters 3, 459 (1979).

6. T. de Jong, Z. L. Wang and F. W'. Saris, Phys. Letters 90 A3, 147 (1982).

7. K. P. Huber and G. Herzberg, Molecular Spectra and Molecular Structure (van Nostrand Reinhold Company, New York, 1979), Vol. IV. 
8. M. von Allmen, Fundamentals of energy deposition, in: Laser Annealing of SemiConductors, eds. J. Plate and J. Mayer (Academic Press, New York, 1982).

9. L. L. Chang, in Handbook on Semiconductors, Vol. 3, ed. S. P. Keller (NorthHolland, 1980), p. 570.

10. K. H. Kingdon and I. Langmuir, Phys. Rev. 21, 380 )1923).

11. R. Kasprzak, J. Oliver and R. Poirrier, Vide. Couches Minces 36, 207, 531 (1981). 